\title{
El contexto de descubrimiento y la psicología de la ciencia
}

\author{
Manuela Romo \\ Universidad Autónoma de Madrid

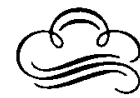

Resumen

Desde que el neopositivista Reichenbach planteó la división, en el análisis de la ciencia, entre contexto de descubrimiento y contexto de justificación se empieza a considerar el primero como una tarea que incumbe a los psicólogos. Aqui se revisan las explicaciones de la psicologia actual sobre el descubrimiento científico que se incluyen dentro de ese nuevo enfoque cientifico propugnado por los postpopperianos, donde convergen el análisis bistórico, sociológico y psicológico. Se reseñan las aportaciones más relevantes para la explicación de la creatividad científica: el estudio cognitivo de casos de Gruber, el análisis temático de Holton, la concepción del genio científico de Simonton asi como las obras uprogramáticas» definidoras de los contenidos y métodos de esta "ciencia de la ciencia» como las de Tweney et alt., Miller o Giere.

\section{Abstract}

Reichenbach separated the science analysis into discovery context and justification context; since then, the discovery analysis is considered as a job for psychologists. Science philosophers lighlight the need of psychology of science. In this paper the contributions of contemporary psychology to scientific discovery included in the uscience of sciencer are reviewed. swuch a dicipline advocated by the postpopperians include bistorical, psychological and sociological aproaches tivity are reviewed: The Gruber's "cognitive study case method, the Holton's «thematic analysis», the Simonton "scientific genius" notion. Finally, the "programatic books» that define contents and methods of this science of science are reviwed.

Dirección de la autora: Departamento de Psicología Básica. Facultad de Psicología. Universidad Autónoma de Madrid. 


\section{0}

\section{DOS DIMENSIONES DE ANALISIS EPISTEMOLOGICO EN LA CIENCIA}

En 1938 uno de los fundadores del positivismo lógico, Hans Reichenbach planteaba, por primera vez, la necesidad de distinguir entre contexto de descubrimiento y contexto de justificación en el análisis de la ciencia, encomendando el primero al trabajo de los psicólogos. Posteriormente escribe:

«El acto de descubrimiento escapa al análisis lógico; no existen reglas lógicas según las cuales pudiera construirse una "máquina descubridora" que asuma la función creadora del genio. Más la tarea del lógico no es dar razón de los descubrimientos científicos; todo lo que le incumbe bacer es analizar la rela. ción existente entre los bechos dados y una teoría que se presente con la pretensión de explicarlos. En otras palabras, a la lógica solamente le importa el contexto de justificación». (Reichenbach, 1951).

A partir de entonces quedarán claramente definidas las diferencias entre un análisis de las teorías científicas como productos acabados y una análisis de la génesis y desarrollo de las teorías. Y, como también deja claro el mismo Reichembach, al filósofo de la ciencia sólo le incumbe lo primero.

Así había sido y así iba a ser un tiempo más, hasta que una nueva generación de teóricos de la ciencia, «alternativos» a todo lo anterior, decidieran que un análisis epistemológico de la ciencia resulta empobrecido si no alcanza a los términos en que ésta se forja dentro del pensamiento del científico.

Pero, centrémonos ahora sobre las formas que ha tenido el justificacionismo en la teoría de la ciencia y las críticas que han hecho limitar su validez explicativa. Pueden definirse en estos dos términos: «inducción» en el positivismo lógico y «falsación» en Popper.

Para Carnap (1932) en la ciencia hay un lenguaje teórico, de naturaleza formal y un lenguaje observacional de constatación de hechos empíricos. De esta forma, sólo existen dos tipos de lenguajes significativos en la ciencia que permiten la contrastación de su verdad o falsedad: formales o fácticos; entre unos y otros se da una independencia, garantía de la objetividad de la ciencia. Considerar que la observación está predeterminada es, para Carnap y los filósofos neopositivistas, caer en el irracionalismo en la explicación científica. Contra esto reivindican la exclusiva inducción pura como fuente de datos.

La ilusión inductivista, sin embargo, tiene raíces profundas pero ha sufrido oscilaciones a lo largo de la historia. Por ejemplo, en Newton tenemos un ejemplo de inductivismo radical. El gran genio de la física moderna se mostraba jactancioso de no necesitar trabajar con hipótesis valiéndose, en muchas investigaciones, de un método de análisis y composición particularmente existoso en sus experimentos de óptica. Admitiendo la necesidad, en algún caso, del uso de hipótesis, es requisitorio que éstas se subordinen a la experiencia, de acuerdo con las reglas definidas por él en los «Principia mathematica» (1687). En el mismo sentido se manifestaría Locke en su «Ensayo sobre el entendimiento bumano» (1690), con más escepticismo aún que Newton sobre lo que entendía como el uso de principios o hipótesis hechas a placer.

En nuestro siglo y ya dentro de una auténtica filosofía de la ciencia, Carnap sienta el valor epistemiológico de la inducción al concebir, como justificación de las proposiciones científicas, la evidencia inductiva.

Pero los argumentos de Popper van a arremeter eficazmente contra la inducción positivista con lo que llama el «mito de la generación de hipótesis inducti- 
vas» (Popper, 1962). Como él mismo señala, se trata del tema del huevo o la gallina:

«El problema "¿qué viene primero, la hipótesis $(H)$ o la observación $(O)$ ?" es soluble; igual que el problema "¿qué viene primero, la gallina $(H)$ o el huevo $(O)$ ?". La respuesta a la última es "Una clase anterior de huevo"; a la primera "Una clase anterior de bipótesis". Es absolutamente cierto que, cualquier bipótesis particular que eligamos la babremos precedido de observaciones, que hay que explicar. Pero esas observaciones, por su parte, presuponen la adopción de un marco de referencia, un marco de expectativas: un marco de teorias” (Popper, 1978: 47).

Para Popper, pues, no existen observaciones puras, libres de teoría. Las proposiciones científicas no pueden ser justificadas por evidencia inductiva. Si las prescripciones de Locke y Newton limitaban el uso de hipótesis, las de Popper sólo exigen que éstas sean testables - ya veremos en qué forma- y no faltará quién anule, incluso, ese requerimiento. (Feyerabend, 1975).

¿Hipótesis u observaciones? Las críticas al positivismo no suponen tampoco el destierro definitivo de la inducción. Que sepamos, nadie ha podido demóstrar la validez excluyente de una u otra postura. Así, por ejemplo, si las leyes de la óptica de Newton parten de observaciones puras, sin embargo, en sus leyes del movimiento hace claramente recurso de constructos hipotéticos. Explican y predicen el comportamiento de las cosas, sean planetas o manzanas que caen pero, en sí mismas, se refieren a eventos que no pueden ser observados. (Butss y Davis, 1970).

El problema es demasiado complejo y se hace necesaria investigación empírica dentro de una cienca de la ciencia - luego hablaré de ello- para definir cuándo, cómo y qué hipótesis se usan exitosamente. Pues parece que hay circunstancias donde es más útil el uso de hipótesis especulativas y también que hay una interacción entre la clase de hipótesis y la clase de situación para determinar qué sería lo óptimo. (Tweney et alt., 1981).

Pero, volviendo al positivismo lógico, hay que concluir que no ha dado una descripción válida de la ciencia ni tampoco ha cambiado la actividad de los científicos. En la misma física, Einstein ha arremetido en diversas ocasiones (1949, 1959) contra la validez de la inducción y otros preceptos positivistas como la axiomatización y el operacionismo como justificación de las teorías científicas. Nuestra joven ciencia psicológica, sin embargo, se formaría en las normas epistemológicas de los filósofos del círculo de Viena en el desarrollo del primer paradigma: el conductivismo.

A la caida del positivismo lógico, la nueva versión de la filosofía de la ciencia, dominada por Popper da el salto de la experiencia a la razón, como criterio de justificación de las teorías.

Esta nueva teoría de la ciencia que se ha llamado «racionalismo crítico» ha tenido y tiene un enorme peso; pero, veamos las coordenadas de esta posición racionalista: Popper mantiene en común con sus predecesores un interés absoluto en el contexto de justificación. El trabajo del filósofo es ofrecer, mediante un análisis de orden lógico exclusivamente, normas a los científicos para guiarles epistemológicamente en las formas de alcanzar una ciencia verdadera. La justificación de las proposiciones científicas, en vez de remitirse al dato empíri$\mathrm{co}$, como pretenden los neopostivistas, debe remitirse al principio lógico de la falsación, considera Popper desde su firme postura de criticismo lógico.

Está claro que una concepción normativista tal es excluyente del análisis psicológico. Poper rechaza para la psicología el jugar cualquier papel en el análisis 
del conocimiento científico, cuya naturaleza considera completamente diferente de la del conocimiento ordinario. Esto impide cualquier posibilidad de incluir en el análisis epistemiológico el «contexto de descubrimiento», lo que choca frontalmente con las posturas de sus contemporáneos, paradójicamente llamados postpopperianos, contra Kuhn, Hanson o Feyerabend que toman, como veremos, una actitud más abierta - donde cabe el contexto de descubrimiento- e interdiciplinar - donde cabe la psicología o la sociología - en el análisis de la ciencia.

La falsación popperiana tiene su precedente en Descartes, también poseedor de una actitud epistemológica racionalista que le lleva a hacer contribuciones a la filosofía y a la matemática. Pero, la duda metódica cartesiana, sólo ofrece como punto de partida para la construcción de una ciencia física unos axiomas «claros»y «distintos» pero no sometibles a verificación empírica.

A lo que si dio pie esta duda ontológica fue al interesante debate sobre las fuentes últimas del conocimiento, abandonado desde Hume y no resuelto. Precisamente, en otro nivel de análisis, tal debate sigue abierto para la psicología cognitiva actual y produce no pocas polémicas y quebraderos de cabeza cuando se entra en el tópico de la representación del conocimiento. La penosa tarea de explicar cómo han llegado hasta ahí esos contenidos ya tomen la forma de «esquemas», «categorías", «proposiciones», «nodos» o «eslabones» o cualquier otro constructo es un terreno resbaladizo donde es siempre preferible «escurrir el bulto», sobre todo cuando los modelos proceden de la inteligencia artificial (Schank y Abelson, 1977; Rumenhart y Ortony, 1977; Anderson, 1976, 1983; Smith, Soben y Rips, 1974; Collins y Quillian, 1972, ...) pero también cuando se trata de formulaciones tan «ecológicas» como la de las categorías naturales de Rosch $(1973,1975)$.

Pero, volviendo a la duda, sin embargo parece haber sido más productivo para el desarrollo de la ciencia que se convirtiese en operativa aunque nos haya dejado sin resolver - ¿es que acaso se puede? - el tema de las fuentes últimas del conocimiento.

Hay una tradición anterior a Popper en el siglo XviII con Hartley, en el sentido de no considerar verdaderas las hipótesis científicas hasta que no hayan superado la prueba de la falsación. Pero, hasta Popper no se convierte la desconfirmación en el núcleo central de una teoría de la ciencia.

Sólo la falsación o desconfirmación nos permite, según Popper, diferenciar entre ciencia y pseudociencia. Debemos sospechar de una teoría que ofrece muchos datos confirmatorios pero, sin embargo, no hace predicciones falsables; porque por muchos confirmatorios que aporte una proposición científica, una sola desconfirmación es suficiente para falsearla. Existe una asimetría lógica entre la evidencia confirmatoria y desconfirmatoria, lo que es evidente en las proposiciones enunciadas con la fórmula del condicional «si p entonces q».

Aunque la influencia del método de desconfirmación de Popper se ha dejado sentir en la Psicología, entre otras cosas, con la descalificación del psicoanálisis, acusado por Popper $(1962,78)$ de pseudociencia; sin embargo, cada vez son más las voces que se alzan contra la realidad de la falsación como método.

Los pioneros trabajos de Wason $(1966,68)$ sobre el uso del condicional y todos los que le han seguido han demostrado que es muy frecuente el error de acudir a la confirmación más que a la falsación en el uso del condicional y que, por otra parte, una teoría científica poderosa bien confirmada no es abandonada porque aparezca una anomalía de este tipo, en cuyo caso el científico acude a modificaciones expostfacto. 


\section{3}

Parece que las prescripciones de Popper podrían traducirse a la práctica con un método de trabajo de hipótesis múltiples, como ha hecho Eccles en la neurofisiología o Watson y Crick en el descubrimiento de la estructura molecular del DNA. Pero, de cualquier manera, es inexcusable una calificación psicológica de contraintuitivas (Tweney et alt. 1981) a unas conclusiones donde no cuenta la confirmación, la inducción no juega ningún papel en la ciencia y la tarea de los científicos consiste en refutar sus propias hipótesis.

\section{LA EVOLUCION DE LA FILOSOFIA DE LA CIENCIA. DEL CONTEXTO DE JUSTIFICACION AL CONTEXTO DE DESCUBRIMIENTO}

A partir de los años 60 las obras de autores como Kuhn, Hanson, Lakatos o Feyerabend dominan el panorama de la teoría de la ciencia; especialmente es de destacar el impacto de «La estructura de las revoluciones científicas» de Kuhn (1962). El resultado es un modelo de ciencia más dependiente en su explicación de sus hacedores - diría yo-. Los filósofos se percatan explícitamente de que la ciencia es una actividad humana y ello significa que, como cualquier otra cosa que haga el hombre, tiene unos condicionantes externos, unos condicionantes de carácter social y psicológico. La imagen del científico será, a partir de ahora, completamente distinta, más parecida a un hombre o mujer. Ese singular personaje incondicionado que hace observaciones puras o valora con una razón fría sus producciones tiene que abandonar el ilusorio lugar donde se pueden hacer tales cosas, para estar en el mundo haciendo ciencia como otros hacen otras cosas.

Incluso los propios científicos parecen sentirse más a gusto en el mundo que en su torre de marfil. Ha sido muy frecuente su reivindicación del contexto de descubrimiento, aunque la misma institución científica esté organizada de tal manera que minimiza la importancia de estos elementos. El tópico de la ciencia como actividad compartida se asocia a un desprecio sistemático de lo que Einstein llamó la «lucha personal». El vacío que la Psicología ha dejado durante mucho tiempo en el análisis de las condiciones psicológicas del descubrimiento científico lo han intentado llenar los propios científicos con muy bonitos retratos introspectivos de gran valor heurístico para la psicología de la creatividad científica y, claro está, para el campo científico específico de cada uno. Ahí están, por ejemplo, la «Psicología de la invención en el campo matemático» de Hadamard (1945), las reflexiones autobiográficas de Poincaré en «Los fundamentos de la ciencia» (1913), de Einstein en «Nota autobiográficas» (1949), la «Biología de la invención» de Nicolle (1932) o el relato de Watson sobre su encuentro con la doble hélice (Weisberg, 1986).

Se ha desmoronado la torre de marfil que guardaba al científico y el resultado ha sido ventajoso para todos. Pero, la ventaja principal - a mi entenderson las posibilidades que se han abierto a la indagación sistemática y, ya nunca más timorata del pensamiento científico. Como dicen Tweney et alt.:

"Como el acto de creación artística es visto por mucbos como inefable e incognoscible, así también la indagación científica ba sido vista como misteriosa y dejada sin explorar» (1981:2).

Los psicólogos han ignorado los temas relativos al conocimiento científico, salvo casos aislados. No se ha enfocado la cuestión central ¿cuáles son los mecanismos 
cognitivtos que subyacen al pensamiento científico? Tweney, Doherty y Mynatt dan una respuesta contundente en su libro: «La actividad cognitiva constituye el corazón de la actividad científica» (1981: 2).

Como se deja entrever en lo que acabo de decir, una de las consecuencias derivadas del nuevo modelo de ciencia será que se empiece a considerar la propia ciencia como objeto de análisis científico más que filosófico. Y no sólo, como veremos, para la ciencia psicológica.

El purismo ya empirista, ya racionalista de las versiones del positivismo lógico y de Popper desaparece del panorama epistemológico frente a las versiones de estos filósofos llamadas por Suppe (1974) «posiciones alternativas».

El énfasis que va a tomar el contexto de descubrimiento se ubica en un marco más amplio de cambio en la explicación de la ciencia desde lo interno a lo externo y que vamos a considerar primero.

\subsection{Lo externo versus lo interno}

Una acertada valoración de la historia real de la ciencia, según Kuhn, nos lleva a considerar la importancia de los factores externos, de las «weltanschaungen» determinantes de lo que se investiga y cómo se investiga, frente a un análisis internalista que aisla a la ciencia de todo, - hasta del sujeto que la hacey que lleva a la errónea conclusión de que la ciencia es acumulativa. Concepción que es incompatible, según Kuhn, con la historia real de la ciencia.

Frente a la estática visión anterior, ahora se señala el carácter dinámico de la ciencia. Esta no es acumulativa, sino que evoluciona sufriendo crisis en su desarrollo y en un proceso dialéctico donde, a un período de ciencia normal sucede un período de ciencia revolucionaria que da pie a la aparición de otro período de ciencia normal (Kuhn, 1962). Las revoluciones constituyen episodios en la historia de la ciencia donde un paradigma es sustituido por otro.

Este panorama dialéctico que, desde una interpretación estática y estructural de la ciencia, podría parecer un frenético baile choca frontalmente contra la actitud positivista pero es también capaz de incomodar a algún postpopperiano: es el caso de Lakatos. Todavía defensor de un justificacionismo y denostador de lo interno, rechaza el cariz de irracionalismo que una visión tal de la ciencia lleva implícito, lo que cuadra con su fidelidad al racionalismo de Popper, (Lakatos, 1978).

Más escandalizadora que la de Kuhn sería la postura de Feyerabend, de claros vitores al irracionalismo. En este autor tenemos ya una auténtica declaración en contra de una filosofía de la ciencia. Para él no tiene sentido una epistemología con carácter prescriptivo general -éste es precisamente uno de los elementos que configuran la situación epistemológica actual-. Si hay algún principio general válido para este autor es el de que «todo sirve». No tiene sentido dictar criterios generales para la resolución de problemas científicos cuando, de los análisis individuales resulta que los científicos tratan con los problemas guiándose de su experiencia. Además, Feyerabend concede especial relevancia a la intuición relacionando el saber científico con las artes y las humanidades. Su anarquista posición se enfrenta contra un análisis lógico y prescriptivo, «contra el método», como reza su obra más conocida (Feyerabend, 1975).

Pero, si lo que acabamos de decir socava la imagen ideal de la ciencia de los filósofos precursores, la definición que hace Kuhn de la actividad de los científicos en los períodos de ciencia normal contribuye a su definitorio derrumbe: cuando un paradigma se hace dominante, los científicos comparten una matriz 
disciplinar común que funciona prescriptivamente hasta en la conformación de un lenguaje observacional -queda muy lejos la invarianza de las observaciones-. Esto predetermina también unos sesgos confirmatorios y el rechazo de las anomalías. Si no se pueden incluir éstas en la matriz disciplinar, se responsabiliza al científico o a los instrumentos experimentales. En resumen, en los períodos de ciencia normal la actividad científica es rutinaria pero, a la vez, dogmática; en este sentido, se puede hablar de una dictadura epistémica en los paradigmas. En suma, las teorías no se abandonan porque aparezcan unas cuantas anomalías. Con este contexto si es congruente lo que dice Lakatos de que no hay refutación sin una teoría mejor. Una teoría no se abandona si no es para ser sustituida por otra:

«El criterio de Popper ignora la extraordinaria tenacidad de las teorías científicas. Los científicos son duros de pelar. No abandonan una teoría sólo porque los bechos la contradigan. Inventan una bipótesis rescatada para explicar lo que llaman entonces una sencilla anomalía o, si no pueden explicar la anomalia la ignoran y dirigen directamente su atención a otros problemas. Nótese que los científicos hablan de anomalias, ejemplos, recalcitrantes, no de refutaciones». (1978: 3).

Parece que esta imagen del científico recalcitrante, aferrado rígiidamente a sus teorías y sordo a las críticas tampoco cuadra con la imagen ideal de fidelidad a la observación objetiva o a la razón que somete a duras pruebas las porpias teorías.

\subsection{Contexto de descubrimiento versus contexto de justificación}

También en conexión con lo anterior toma fuerza lo externo en la explicación del hecho científico, al anteponer el análisis del contexto de descubrimiento al mero justificacionismo anterior de enunciados científicos. Externo, si es que lo externo es apelar a las condiciones piscológicas desencadenantes de los procesos de pensamiento científco.

Ya hemos visto que Reichenbach es el primero en hacer la distinción formal entre contexto de justificación y contexto de descubrimiento aunque rechaza el análisis de este por la lógica. Hanson, sin embargo, ha considerado que sí cabe una filosofía del descubrimiento que desarrolla en su obra «Patterns of discovery» (1958). Su idea es que el descubrimiento se produce dentro de patrones o esquemas teóricos que orientan la investigación y buscan su propia confirmación. Ahora bien, el tipo de cuestiones que pueden plantearse sobre este tópico requieren más de un análisis empírico que filosófico.

No es suficiente con un análisis lógico de productos acabados. Después de todo lo que llevamos visto, está claro que el pensamiento científico no se rige por las reglas de la lógica; es más, de hecho es a menudo, ilógico. Estudiar el proceso de gestación de los descubrimientos se considera actualmente una gran contribución a la comprensión del pensamiento científico pero, también es cierto que en ello se impone un análisis psicológico.

Holton, que habla de acontecimientos para referirse a los productos del trabajo científico explica así el aspecto más personal de un acontecimiento tal:

«Nos situamos aqui en el contexto de descubrimiento, tratando de comprender «el momento del alumbramiento» que puede estar poco documentado y que no tiene por qué ser necesariamente apreciado o comprendido por el mismo autor. Con excepción de los trabajos sobre contadas figuras, como Kepler o Einstein, los científicos (y los filósofos) ban sido bastante poco comprensivos con este tipo de estudios» (1982: 20). 


\section{6}

Ha llegado pues, la hora de ocuparse del «momento del alumbramiento» pero desde una perspectiva completamente nueva y ya no filosófica, donde han de converger distintas disciplinas en el estudio científico que, en lo que dice al contexto de descubrimiento, requiere del psicólogo pero también del historiador de la ciencia, como el mismo Holton hace en la física. Pero, ello es asunto de esa nueva disciplina de la que trataré a continuación: la ciencia de la ciencia.

\section{UNA NUEVA DISCIPLINA: LA CIENCIA DE LA CIENCIA}

Las vicisitudes experimentadas en su evolución por la teoría de la ciencia, las corrosivas formulaciones que acabamos de ver contra la propia epistemología filosófica (de Kuhn o Feyerabend) junto con las aprotaciones, cada vez más sólidas, al análisis del pensameinto científico hechas por los psicólogos, constituyen los tres condicionantes que han abocado a un estado crítico de la filosofía de la ciencia; donde la única salida viene, de alguna manera, predeterminada por los propios desencadenantes de la crisis. Desencadenantes que han intervenido como tres causas interdependientes. Pero, vayamos por partes:

1. Por una parte, los míticos principios de la gran escuela del positivismo lógico resultaron un estrepitoso fracaso no ya por la ignorancia que las ciencias tuvieron de los mismos - a excepción de la primera psicología conductivista que no los ignoró y también fracasó- sino por las propias críticas internas de los filósofos. El «ideal de unidad de la ciencia» se quedó en eso, en ideal y sus otros principios básicos, como hemos visto, se convirtieron en el «dogma de la invarianza» o en el «mito de la inducción». Pero también ha hecho aguas la filosofía racionalista de Popper porque, en última instancia, la falsación también es un mito, como los postpopperianos se encargan de demostrar apelando a la historia real de la ciencia o los psicólogos apelando a la vida real o al funcionamiento cognitivo del científico.

2. El panorama presentado por los postpopperianos y dominante en la década de los setenta, tiene unas connotaciones que piden a gritos la salida por un nuevo enfoque: abandono de una teoría general del método como propone Feyerabend, papel determinante de lo social y lo psicológico en el nuevo análisis de la ciencia, acudir a la historia real de la ciencia aunque el resultado nos avoque a tener que asumir ese irracionalismo tan detestado por Lakatos y el adiós al justificacionismo sustituido por el interés en el contexto de descubrimiento.

3. En cuanto a lo psicológico, el área de influencias se divide en dos tópicos: los estudios sobre inferencias que ponen en entredicho la supuesta lógica en el decurso del pensamiento racional no sólo del hombre de la calle, sino también del científico, en trabajos de autores como Wason, Johnson-Laird, Kahneman y Tversky, etc,.. y los estudios recientes sobre la creatividad científica que, bien empleando experimentos de mayor o menor validez ecológica o bien aplicando versiones del método clínico piagetiano a figuras históricas, están aportando conocimiento al contexto de descubrimiento (Giere, 1989; Gruber, 1981; Gruber y Davis, 1988; Holton, 1972, 78, 82; Mittrof, 1974; Mynatt, Doherty y Tweney, 1978; Newell y Simon, 1972; Simonton, 1987, 88; Sternberg, 1988; Weisberg, 1986) sin excluir por su valor testimonial y heurístico las introspecciones de científicos como Einstein, Poincare y otros. El famoso librito de Ghiselin, «El proceso creativo» (1952) es una verdadera joya por el valioso material introspectivo que presenta de científicos y también de artistas ${ }^{1}$. 
Trato relevante de lo histórico y del contexto social en el devenir de la ciencia, demanda cada vez mayor de una epistemología de matices psicológicos... ¿qué hacer con todo esto? Son datos que rebasan el marco de un análisis puramente lógico; la respuesta parece estar en una aproximación interdisciplinar y de carácter empírico que se ha dado en llamar «ciencia de la ciencia».

La nueva visión de la ciencia se distancia del tradicional análisis filosófico que la ha mirado siempre como un producto acabado. No se puede minimizar el papel que, desde la revolución científica, ha tenido el conocimiento científico en la producción de cambios sociales y políticos. La ciencia ha cambiado la visión del universo y de la propia vida humana.

Por otra parte, no es nuevo el interés por hacer una ciencia de la ciencia; lo que si es nuevo es la viabilidad de su estudio por métodos experimentales. Así, cuando en el estudio del pensamiento científico se adopta tal enfoque surge un largo set de cuestiones susceptibles de tratamiento empírico: ¿cómo se formulan las hipótesis? ¿cuándo se abandonan? ¿cuándo no se abandonan? ¿existen limitaciones cognitivas para la actividad del científico? La psicología actual está preparada para responder este tipo de cuestiones y sólo a partir de los principios emanados de esta psicología de la ciencia se abre la posibilidad de una epistemología empírica. El libro de Tweney, Doherty y Mynatt, «On scientific thinking» es un afortunado intento de exposición de las cuestiones relevantes que esta nueva disciplina debe abordar.

Pero, aunque sólo a partir de ahora se haya hecho experimental, el interés en la psicología de la ciencia no es nuevo. Desde Bacon, al comienzo de la revolución científica, muchos filósofos y científicos se han percatado de que entender los procesos mentales humanos resulta propedéutico para en entendimiento de la ciencia.

Aunque el conocimiento científico daría al hombre un nuevo poder sobre la naturaleza, para el desarrollo de ese conocimiento es esencial entender la naturaleza humana. Así lo supo ver Bacon en su «Novum organum» (1620) que llegó incluso a predecir algún tema esencial para la psicología de la ciencia, como el sesgo confirmatorio en la inferencia.

Posteriormente, las versiones sobre el papel de la psicología en la explicación del conocimiento científico han sido diversas. Por una parte, la actitud del fundador del positivismo, Comte, que fue de claro rechazo a la psicología como algo ajeno al mundo de las ciencias es heredada por el fundador de la psicología científica, Wundt, quién adoptando una posición empirista para el análisis de los contenidos mentales niega, sin embargo, que la experimentación puede extrapolarse hasta el análisis del pensamiento científico. De la misma manera, pero por razones distintas, como hemos visto, rechaza Popper el papel de la psicología en el entendimiento de la ciencia.

De otra parte, arranca de Mach (1905) un precedente sólido de la psicología de la ciencia, al destacar la importancia que para el propio científico tiene el conocimiento de los procesos por medio de los cuales, obtiene y extiende su conocimiento. Lo más interesante en la concepción de Mach, al respecto, es su formulación metodológica del análisis del pensamiento, sobre todo si la comparamos con los puntos de vista mantenidos por los psicólogos contemporáneos - acabamos de citar a Wundt-. Para Mach la psicología es, sencillamente, equiparable a la física; tiene los mismos métodos. Para analizar el pensamiento no hace falta ir más allá de lo empíricamente dado. Tanto la vida física como la mental se explican a partir de los hechos de la vida observable. Por otra parte 
¿qué otra cosa podría decir un autor a quien se considera padre del positivismo lógico?

Finalmente, en Mach tenemos también otro gran precedente de los contenidos y el enfoque de la actual psicología de la ciencia, en el sentido de considerar a ésta como una extensión del pensamiento ordinario. Tal ha sido un principio mantenido en la epistemología genética de Piaget y la escuela de Ginebra (Piaget, 1950; Inhelder y Piaget, 1958; Karmiloff-Smith e Inhelder, 1975) donde se ha abordado el estudio empírico del desarrollo de los conceptos científicos en niños y adolescentes.

Sin llegar a la ironía de Holton (1982) de considerar a la ciencia una actividad pedestre que, sin embargo, ha reportado grandes éxitos a la humanidad; lo que sí es cierto, es que el asumir esa continuidad y rescatar del mundo de lo misterioso la creatividad científica permite abordar su estudio de la piscología cognitiva; y, a lo que se ve, con éxito. Așí, para Herbert A. Simon (1977) no existen diferencias cualitativas entre el trabajo consciente y no consciente - la tal manida «incubación»- en la solución de problemas científicos, entre el trabajo altamente creativo y el trabajo diario del científico, entre los procesos de ciencia normal y revolucionaria. Ciertamente, en las recientes versiones de la psicología cognitiva sobre la creación científica: Gardner, Sternberg, Perkins, Weisberg, Giere,... se entiende ésta como una extensión de los procesos de pensamiento ordinario (Romo, 1990).

Quiero acabar este asunto de la importancia dada por los estudiosos de la ciencia a la psicología citando a Kuhn, con el cual esta tradición cuaja definitivamente en la filosofía de la ciencia y, a partir del cual, se hace ineludible una psicología de la ciencia. Según él, hay muchas cuestiones de especial relevancia para el conocimiento científico que no han tenido satisfactoria respuesta. Así, en 1970 se refería a:

- Si aumenta el número de puntos de vista incompatibles al crecer el número de comunidades de especialistas.

- Por qué causas abandonan los científicos el ideal de unidad de la ciencia:

- Aunque parece que en cada momento el conocimiento abarca lo que hay que conocer, dejando sólo visibles rompecabezas en el horizonte del conocimiento existente, podríamos plantearnos si los científicos contemporáneos conocen más o menos de lo que hay que conocer de su mundo que sus predecesores de otras épocas.

Hasta que no sepamos dar contestación a cuestiones como éstas no sabremos explicar qué es el progeso científico. $Y$ añade Kuhn que, tan sólo conociendo lo que valoran los científicos podremos entender qué problemas acometerán; el estudio de la ciencia debe dar una descripción de un sistema de valores y de las instituciones a través de las cuales este sistema se transmite y refuerza. En última instancia, la explicación debe ser psicológica y sociológica (Kuhn, 1970).

Vayamos pues al comentario de los otros enfoques, histórico y sociológico, que convergen en esta nueva singladura del análisis de la ciencia.

La sociología de la ciencia es una disciplina que cuenta con cuarenta años de existencia y su explicación del hecho científico aunque, ciertamente permita obtener alguna respuesta válida a todas estas preguntas, hasta el presente ha tenido poca interacción con el enfoque psicológico. Entender la ciencia como institución social supone que el conocimento científico es un hecho de origen social 
evaluado por un contexto social. Nace esta disciplina como una opción de aplicar los conceptos y métodos de la sociología al entendimiento de la ciencia (Merton, 1957). En sus temas se trata con una especie de científico idealizado como producto social y se ignora el papel del científico como individuo: el «homo científicus» es su objeto frente al individuo científico del enfoque psicológico.

Otra cosa sucede con la historia de la ciencia. Disciplina que nace en forma de trabajos biográficos $\mathrm{o}$ análisis diacrónico de un particular concepto científico $^{2}$ y que sufre un gran vuelco a partir de Kuhn para pasar de la mera descripción al intento de explicación teórica de su objeto mediante la adopción de marcos teóricos de referencia. Así la explicación paradigmática se centra en el cambio científico y las fuerzas que lo producen; y es precisamente, en el carácter psicológico y sociológico que Kuhn atribuye a esas fuerzas donde se halla la confluencia con la psicología.

De una parte, la historia de la ciencia puede ofrecer muchos datos y muy relevantes al que analiza los procesos del pensamiento científico pero, necesita de la seguridad de los fundamentos psicológicos para construir sus teorías (Tweney et alt. 1981). El debate sobre la imaginación científica es un ejemplo muy demostrativo de esto; la exclusiva remisión al dato histórico nos permite considerar que los afortunados insights de los grandes científicos se deben a una accidental combinación de elementos (James, 1880; Mednick, 1962) tanto como a una función metafórica aplicada a un dominio de hechos. Tan sólo bajo una metodología empírica que no tiene cabida en el enfoque histórico-descriptivo pueden someterse a manipulación los fenómenos a estudiar y definirse los principios generales que subyacen a tales fenómenos. El debate sobre la imaginación científica así como otras muchas cuestiones ambigüamente definidas por el método histórico tendrán su solución por una convergencia con el enfoque experimental psicológico. Da fe de ello lo que resta del artículo.

Holton practica esta convergencia y voy a referirme a él por la especial relevancia que tiene para esta ciencia de la ciencia la singular aportación de lo que él llama «análisis temático».

Gerald Holton $(1973,78,82)$ se propone con su trabajo aportar conceptos y métodos «para comprender mejor la manera de pensar del científico que se halla involucrado en el acto de hacer ciencia». (1982: 9).

Su objeto ha sido la física y en su análisis del proceso de gestación de los descubrimientos científicos comienza cotejando la información de los trabajos científicos publicados con la documentación de primera mano ya sean cartas, cuadernos, entrevistas. Propone una flexibilidad en cuanto al método tanto en lo que hace a esa visión ecléctica interdiciplinar del objeto de que hablaba Kuhn (1977) como de lo oportuno de dejarse guiar por la inducción con estas palabras:

«La búsqueda de modelos del pensamiento científico debe ser necesariamente inductiva y empirica en gran parte. Debemos obligamos a la precisión bistórica y al estudio cauteloso basado en las pruebas disponibles. Pero nuèstra buisqueda también debe tener la libertad e imaginación necesarias para producir nuevas herramientas conceptuales con las que se puedan estudiar áreas restringidas como ia que se refiere al funcionamiento de la mente de los científicos (...). Dado que solamente estamos en los comienzos del trabajo de recopilación de los elementos a partir de los cuales pueden diseñarse teorías sobre el pensamiento cientifico, debemos desconfiar de todo esquema que nos ofrezca conclusiones de total certeza». (1982: 16).

Pero, vayamos a la definición de los «themata», su principal aportación, constructo considerado de enorme valor heurístico para la expliación histórica de la ciencia. Se trata, ciertamente, de temas que funcionan como profundas pre- 


\section{0}

concepciones adquiridas durante el proceso de formación del joven científico y que intervienen de forma implícita ayudando y obstaculizando su labor.

A partir de esta definición, habrá a quien le sugiera otros constructos como el de «teoría implícita» o el concepto formal de «metapostulado». Ciertamesnte no se trata de teorías implícitas porque, en la concepción de Holton, no parecen poseer el desarrollo y la articulación proposicional que tiene la teoría implícita (Wegner y Wallacher, 1977; Forgas, 1981). Sí comparten ambos el carácter selectivo, organizador de la información y prescritivo en la acción. La teoría implícita se contrapone a las teorías explícitas de los científicos. Los themata estarían «detrás» de las teorías científicas.

Pero, tampoco funcionan como metapostulados, si entendemos estos siguiendo a De Vega (1984) como «presupuestos conceptuales o prejuicios implícitos en las teorías». En tal caso, la diferencia, pienso que radica en el nivel de generalidad de un concepto y otro. Usando la terminología de Rosch diríamos que, en este contex to de conceptualización epistemológica, los themata ocuparían el lugar más genérico de categorías supraordenadas aunque también funcionando como un sistema de creencias o presupuestos conceptuales implícitos. Así, el metapostulado diverge según el paradigma o incluso, en teorías diversas dentro del mismo paradigma; por ejemplo, la analogía del ordenador en el paradigma cognitivo y los postulados biologistas o constructivistas en Piaget. El thema, sin embargo, tiene un significado mucho más amplio y también, mucho más ambiguo.

En el científico la estructura temática desempeña un papel determinante en la iniciación y aceptación de las ideas científicas o las controversias que puedan establecerse en torno a ellas. Otra nota definitoria del análisis temático es que permite descubrir en la ciencia estructuras relativamente estables que se mantienen a través de las revoluciones y que, incluso, engloban teorías consideradas rivales.

Holton postula que en el científico creativo puede funcionar con un número reducido de themata y que, a los debates subyacen muchas veces diadas antagónicas o a veces tripletes temáticos como:

\section{atomicidad / continuo simplicidad / complejidad \\ constancia / evolución / cambio catastrófico}

Tomenos, para ejemplificar, un caso muy bonito que surge en los albores de la revolución científica y permanece en la física actual. Se trata de los themata de simplicidad y necesidad:

Quizá la valoración sociológica en términos estéticos que Kuhn (1957) considera como causa de la trascendencia de la revolución copernicana pueda tener que ver con la aparición, por primera vez en la ciencia, de los themata mencionados que son, para Holton, la razón primordial de que el modelo heliocéntrico de Copérnico comenzara a dominar en la ciencia moderna. La ciencia, que empieza a imponerse sobre los demás sistemas que se ocupan de la realidad.

La fuerza de la solución copernicana en la explicación de los movimientos de los planetas radica, según Holton, en «lo restringido de su carácter». Nada hay arbitrario, todo está integrado en un todo donde cada elemento: orden, tamaño, movimiento de los planetas, no es más que lo que debe ser. Efectivamente subyace un principio de necesidad que encaja cada detalle al diseño total.

Para Holton existe, de manera más o menos soterrada, esta corriente que 
arranca de Copernico y que incluye la complejidad y la necesidad como garantía de verdades más profundas. Y encuentra buen ejemplo de ello en las coordenadas definidoras de la física actual; aunque la mecánica cuantica permite concebir un gran número de sistemas físicos, sin embargo, combinada con la relatividad se traduce en principios extraordinariamente restrictivos. La naturaleza, como dice Weinberg (1973), se las arregla para ser a la vez relativista y cuántica.

Otra aproximación confluyente de lo histórico y lo psicológico que debemos destacar aquí es la que Gruber y Barret dieron a la luz en 1974 con su trabajo sobre la figura de Darwin titulada «Darwin sobre el hombre: un estudio psicológico de la creatividad científica» y que ha sido posteriormente desarrollada por Howard E. Gruber y su equipo, ya bautizada como «enfoque cognitivo de estudio de casos». Nos ofrecen como resultado la publicación de diversos trabajos que amplían sus análisis más allá de la figura del autor de la teoría de la selección natural y, además, no se reducen a estudiar científicos sino otros personajes de genio creador indiscutible como Benjamín Franklin, William James, John Locke o Dorothy Richardson (véase Gruber y Davis, 1988).

Al igual que Holton, parten de recopilar un materal empírico de pruebas documentales de la historia vital de los personajes en su contexto que, desde su enfoque cognitivista son consideradas como protocolos de solución de problemas de larga duración. A este respecto es interesante observar el desmenuzado y escrupuloso análisis que hace Gruber, en su libro sobre Darwin, de los cuadernos de notas escritos por el naturalista entre 1937 y 1939, previos a la publicación de «El origen de las especies», como argumentación para el desarrollo de su teoría.

En enfoque cognitivo de estudio de casos es una versión constructivista piagetiana para explicar el desarrollo de un teoría en un científico que no es sino la dinámica de unas estructuras de conocimiento que se reelaboran en la interacción con el medio debido a la experimentación y su consiguiente modificación. Este proceso mantiene tanto la coherencia del sistema como el ajuste a los datos de la observación en una doble dialéctica: externa (sujeto-objeto) e interna (relaciones entre ideas e hipótesis). Con ello Gruber minimiza el papel de las iluminaciones repentinas del proceso creador; esos ;ajas! en que han hecho tanto énfasis las versiones fenomenológicas. Sin despreciar la importancia de momentos afortunados, donde se organizan patrones rápidamente, consideran que aquellos deben ser valorados en el contexto de una vida de trabajo, de una mente que prepara el camino para ellos (Gruber y Davis, 1988).

En el estudio del trabajo creativo se trata de entender cómo se organiza, cómo se construye una vida de investigación para formar tres sistemas en evolución, que Gruber denomina: organización del conocimiento, organización del propósito y organización del afecto. En lo dicho queda implícita la importancia que se concede a los procesos motivacionales y afectivos para la creatividad. En términos coloquiales diríamos que el secreto del genio, según esta versión, se resumiría en estas cuatro palabras: ¡una vida de investigación!

Finalmente, otra versión recientísima a destacar es la de Dean K. Simonton «Scientif genius: a psychology of science»; 1988). En sentido estricto, la labor de este autor consiste en desarrollar una teoría psicológica del genio científico. Considera los procesos mentales que subyacen al acto creador: intuición, incubación, «serendipidad»; así como los estilos cognitivos y motivacionales definidores, según él, de una tipología del genio. 
Valora la importancia que sobre el desarrollo del potencial creador del científico tienen algunos antecedentes como el ambiente familiar, la educación, la asunción de roles, el «zeitgeist»... Finalmente Simonton sitúa su teoría psicológica en el contexto de otras «metaciencias»: la sociología y la filosofía de la ciencia.

Aunque no sea la perspectiva asumida en este artículo, no sería cabal terminar sin referirse a otra forma incipiente de abordar el análisis de la ciencia: el enfoque de la ciencia cognitiva.

Richard Giere en su obra «Explaining science: a cognitive approach» aboga por este enfoque que, como meta, se plantea alcanzar una teoría cognitiva unificada de la ciencia donde han de integrarse todos los puntos de vista de las ciencias cognitivas. Sin embargo, reconoce que está aún por desarrollar y de hecho, su manual es también básicamente de contenido piscológico.

Otro tanto cabría decir de la obra del historiador de la ciencia Arthur I. Miller. En su obra "Imagery in scientific thuoght» plantea la importancia de alcanzar una explicación teórica sobre la imaginación en el pensamiento científico. Sobre el análisis de los casos de Bohr, Boltzman, Einstein, Heisenberg y Poincare, repasa las explicaciones que, en lo tocante a la producción de imágenes, se han dado desde enfoques psicológicos cognitivos como la epistemología genética o la teoría de la gestalt sin gran éxito, a su juicio. Pero, lo que importa destacar aquí, es el énfasis que también comparte el autor por «... una aproximación multidisciplinar a un problema fascinante en la historia de las ideas: el pensamiento científico» (1985: xii).

Recordemos, como conclusión, que la ciencia es la actividad humana capaz de generar mayor conocimiento, nada menos que la estructura causal del mundo. Y, sea cual sea, el tipo de análisis que prevalezca en el futuro, deberá tener siempre presente que su estudio es una ciencia humana, una ciencia del conocimiento.

\section{Notas}

1. Menor trascendencia han tenido, a estos efectos, las conferencias de la universidad de Utah sobre la creatividad científica celebradas en 1955, 57 y 59 (véase, Taylor y Barron, 1963), porque su enfoque fundamentalmente psicométrico no se centró en una definición conceptual del pensamiento científico.

2. Como la historia del DAN escrita por R. Olby en «The path to the double helix» (1974).

\section{Referencias}

ANDERSON, J. R. (1976): Language, memory and thought. Hillsdale, Nueva Jersey: Lawrence Erlbaum Associates.

Anderson, J. R. (1983): A spreading activation theory of memory. Journal of Verbal Learning and Verbal Behavior, 22, 261.295.

ButTs, R.E. y Davis, J. W. (Eds.). (1970): The Methodological Heritage on Newton. Toronto: University fo Toronto Press.

Carnap, R. (1932). The elimitation of metahysics thorugh logical analysis of language. En Ayer, A. J. (Ed.): Logical positivism, Glencoe, I11., Free Press, 1959. 
BARRON, F. Y TAYLOR, (1963): Scientific creativity: its recognition and development. Nueva York: Wiley.

Coluins, A. M. y Quilitan, M. R. (1972): How to make a language user. En Tulvin, E. y Donalson, W. (Eds.): Organization of memory. Nueva York: Academic Press.

Crovitz, H. F. (1969): Galton's Walk: Methods for the Analysis of Thinking, Intelligence and Creativity. Nueva York: Harper and Row.

Einstein, A. (1949): Autobiographical notes. En Schilpp, P. A. (Ed.): Albert Einstein: Philosopher-Scientist. Nueva York: Tudor.

Einstein, A. (1959): Remarks on the essays appearing in the collective volume. En Schilpp, P. A. (Ed.): Albert Einstein: Philosopher-Scientist. Nueva York: Harper.

Feyerabend, P. K. (1975): Against Method. Londres: NLB. Trad. cast.: Madrid, Tecnos.

FORGAS, J. P. (1981): Social cognition: perspectives on everyday understanding. Londres: Academic Press.

GHiseling, B. (Ed.). (1952): The creative process. Nueva Jersey: New American Library.

GIERE, R. (1989). Explaining science: a cognitive approach. University of Chicago.

Gruber, H. E. (1981): Danwin on Man. A Psychological Study of Scientific Creativity. Second edition. Trad. cast.: Madrid, Alianza.

Gruber, H. y Davis (1989): Inching our way up Mount Olympus: the evolving-systems approach to creative thinking. En Sternberg, R. The nature of creativity. Cambridge.

Gruber, H. E. y Barret, P. H. (1974): Darwin on Man. Nueva York: Dutton. (Trad. cast.: Madrid, Alianza.

HadAmard, J. (1945): Essai sur la Psychologie de l'invention dans le domaine mathematique. París: Bordas.

Hanson, N. R. (1958): Patterns of Discovery. Londres: Cambridge University Press.

Holton, G. (1972): Mach, Einstein and the Search for Reality. En Holton, G. (Ed.): The Twentieth-Century Sciences: Studies in the Biography of Ideas. Nueva York: Norton.

HolTON, G. (1973): Thematic Origins of Scientific Thoubgt: Kepler to Einstein. Cambridge: Harvard University Press.

Holton, G. (1978): The Scientific Imagination: Case Studies. Londres: Cambridge University Press.

Holton, G. (1982): Ensayos sobre el pensamiento cientifico en la época de Einstein. Madrid: Alianza.

Inhelder, B. y PIAGet, J. (1958): The Growth of Logical Thinking from Childhood to Adolescence. Nueva York: Basic Books.

James, W. (1880): Great Men, Great Thoughts, and the Environment. Atlantic Monthly, 46, 441-59.

Karmiloff-Smith, A. y Inhelder, B. (1975): If you want to get ahead get a theory. Cognition, 3, 195-212.

Kunn, T. S. (1957): The Copernican Revolution: Planetary Astronomy in the Development of Western Thought. Cambridge: Harvard University Press.

KuHN, T. S. (1962): The Structure of Scientific Revolutions. Chicago: University of Chicago Press. Reed. en 1970 con la inclusion de «Postcript-1969».

Kunn, T. S. (Ed.). (1977): The Essential Tension: Selected Studies in Scientific Tradition and Change. Chicago: University of Chicago Press.

LakATOS, I. (1978): The Methodology os Scientific Research Programmes. Cambridge: Cambridge University Press. (Trad. cast.: Madrid: Alianza.

MACH, E. (1905): Knowledge and Error: Sketches on the Psychology of Inquiry. Boston: D. Reidel, 1975. /Ed. Orig. 1905).

Mednick, S. A. (1962): The associative basic of the creative process. En Rothemberg y Hausman (Eds.): The creativity question. Durham: Duke University Press.

Merton, R. K. (1957): Priorities in scientific discovery: A chapter in the sociology of science. American Sociological Review, 22, 635-59.

Miller, A. (1984). Imagery in scientific thought. Boston: Birkhäuser.

Mitroff, I. (1974): Norms and counter-norms in a select group of the Apollo moon scientist. American Sociological Review, 39, 579-05.

Mynatt, B. T., Doherty, M. E. y Tweney, R. D. (1978): Consequences of confirmation and siconfirmation in a simulated research environment. Quarterly Joumal of Experimental Psychology, 30, 395-406.

Neweil, A. Y Simon, H. a. (1972): Human Problem Solving. Englewood Cliffs, N. J.: Prentice Hall.

Nicolle, C. (1932): Biolgie de l'invention. París: Alcan

PIAGET, J. (1950): The Psychology of Inteligence. Routledge and Kegan. (Original francés en Colins, 1947)

Poincare, H. (1913): The Foundations of Science. Nueva York: Science Press. 
Popper, K. R. (1962): Conjectures and Refutations. Londres: Routledge and Kegan Paul, 1978. (Ed. Orig. 1962).

Reichenbach, H. (1938): Experience and prediction. Chicago: University of Chicago Press.

Reichenbach, H. (1951): The Rise of Scientific Pbilosopby. Berkeley: University of California Press.

Romo, M. (1990). Psicología cognitiva y conocimiento científico. Revista de Psicología General y Aplicada, 43, 433-43.

Rosch, E. (1973). Natural categories. Cognitive Psychology. 4, 328-50.

Rosch, E. (1975): Cognitive Representations of semantic categories. Journal of Experimental Psychology: General, Vol. 104, N. ${ }^{\circ}$ 3, 192-233.

Rumelhart, D. E. y OORTony, A. (1977): The representation of knowledge in memory. en Anderson, R. C., Spiro, R. y Montague, W. E. (Eds.): Scbooling and the acquisition of knowledge. Hillsdale, Nueva Jersey: Lawrence Erlbaum Associates.

Schank, R. C. y Abelson, R. P. (1977): Scripts, plans, goals and understanding. An Inquiry in. to buman knowledge structures. Nueva Jersey: Lawrence Erlbaum Associates.

Schneider, D. J. (1973): Implicit Personality Theory: A review. Psychological Boletín, 79, 294-305.

Simon, H. A. (Ed.) (1977): Models of discovery. Boston: D. Reidel.

Simonton, D. K. (1988). Scientific genius. A psycbology of science. Cambridge University Press.

Sternberg, R. J. (Ed.) (1988). The nature of creativity. Cambridge University Press.

SMiTH, E. E., SHOBEN, E. J. y RIPS, L. J. (1974): Structures and processes in semantic memory. A featural model for semantic decisions. Psychological Review, 81, 3, 214-241.

Suppe, F. (1974): The structure of scientific theories. University of Illinois Press, Urbana. (Trad. cast.: Madrid: Ed. Nacional.

Tweney, R. D., Doherty, M. E. y Mynatt, C. R. (Eds.). (1981): On scientific thinking. Nueva York: Columbia University Press.

VeGA DE, M. (1984): Introducción a la psicología cognitiva. Madrid Alianza Psicología.

WASON, P. C. (1966): Reasoning. En Ross, B. M. (Ed.): New Horizons in Psychology. Harmondsworth Middlesex, Penguin.

WASON, P. C. (1968): Reasoning about a rule. Quarterly Joumal of Experimental Psychology, $23,273.81$.

Wegner, D. y Vallacher, R. (1977): Implicit Psychology. an Introduction to Social Cognition. Nueva York: Oxford University Press.

Wegner, D. M. y Vallacher, R. R. (1981): Common-sense Psychology. En Forgas, J. P. (Ed.): Social cognition perspectives on everyday understanding. Academic Press.

WEINBERG, S. (1973): Where We Are Now. Science, 180, 276-78. 